\title{
Gravity in View of the Theory of Orbiting Binary Stars
}

\author{
Stefan L. Hahn \\ Retired Professor, Institute of Radioelectronics and Multimedia Technology, Warsaw University of Technology, \\ Warsaw, Poland \\ Email: st.hahn@wp.pl
}

How to cite this paper: Hahn, S.L. (2018) Gravity in View of the Theory of Orbiting Binary Stars. Journal of Modern Physics, 9, 1954-1969.

https://doi.org/10.4236/jmp.2018.910124

Received: June 7, 2018

Accepted: September 10, 2018

Published: September 13, 2018

Copyright (c) 2018 by author and Scientific Research Publishing Inc. This work is licensed under the Creative Commons Attribution International License (CC BY 4.0).

http://creativecommons.org/licenses/by/4.0/

\begin{abstract}
In this paper, we investigate orbiting of two stars having equal masses. We consider two models: with a circular orbit and with two elliptical orbits having a common center of a mass located in a common focal point. In the case of the circular orbit, we applied the notion of the instantaneous complex frequency. The paper is illustrated with numerous formulas, derivations and discussion of results.
\end{abstract}

\section{Keywords}

Gravity, Inertia, Complex Frequency, Curvature Radius

\section{Introduction}

Three years ago, the author presented a paper describing the gravitational forces as a result of anisotropic energy exchange between baryonic matter and quantum vacuum [1]. Here, we try to show that the theory of circulation of double stars around a common center of mass yields arguments in favor of the above theory. Our goal can be achieved by investigating orbiting of two stars having equal masses. We present two such models: the first one with a circular orbit and the second one with two elliptical orbits with a common center of a mass located in a common focal point. The presented mathematical descriptions of the above models are derived by the author and certainly only the methods of derivations are new. Most of the results belong to the existing knowledge. As regards the circular orbit, we applied the notion of the instantaneous complex frequency. We introduce the following notations:

○ MKS system of units is applied.

○ Ellipse: $a$-semi-major axis, $b=$ semi-minor axis, $\varepsilon$-eccentricity;

○ $s(t)=\alpha(t)+j \omega(t)$-instantaneous complex frequency; 
○ $G=6.67384 \times 10^{-11}\left[\mathrm{~m}^{3} /\left(\mathrm{kg} \cdot \mathrm{s}^{2}\right)\right]$ - gravitational constant;

○ $(\rho, \varphi)$-polar coordinates of the ellipse centered at the focus;

- $\rho_{c}$-curvature radius;

○ $P$-power;

○ E-energy;

○ F-force;

○ $T=$ orbital period.

\section{A Model of a Double Star of Equal Mass Orbiting on a Circular Orbit}

Figure 1 shows a circular orbit of a constant radius $\rho_{0}$. Both stars are separated by the distance $l_{0}=2 \rho_{0}$. The angular position of the first star is defined by the phasor $\psi_{1}(t)=\rho_{0} \exp \left(j \omega_{0} t\right) ; \varphi_{1}(t)=\omega_{0} t$, and the second by $\psi_{2}(t)=\rho_{0} \exp \left(j \omega_{0} t+\pi\right) ; \varphi_{2}(t)=\omega_{0} t+\pi$. This system is described by the equality of two forces: the gravitational force of attraction and the centrifugal inertial force.

For the circular orbit of Figure 1 (no inspiral), the two forces are collinear and have opposite directions. They should have the same magnitude. This equality of forces is described by

$$
\boldsymbol{F}_{g}=\frac{G m^{2}}{4 \rho_{0}^{2}}=\boldsymbol{F}_{c}=m \omega_{0}^{2} \rho_{0} .
$$

We get the following time-independent relations between the angular velocity $\omega_{0}$ and the radius $\rho_{0}$ :

$$
\rho_{0}=\sqrt[3]{\frac{G m}{4 \omega_{0}^{2}}} \quad \text { or } \quad \omega_{0}=\sqrt{\frac{G m}{4 \rho_{0}^{3}}}
$$

The orbital tangential velocity is $v_{0}=\omega_{0} \rho_{0}$. Therefore, the kinetic energy of the system is $E_{k}=m v_{0}^{2}$ and the potential energy $E_{p}=-2 \rho_{0}\left|\boldsymbol{F}_{g}\right|$. The potential energy is negative. Its value equals twice the kinetic energy. Therefore, the total energy of the system is negative and time independent. Several authors derived formulae for calculation of the power of gravitational waves emitted by the system of Figure 1. The derivations apply linearized version of Einstein's theory of relativity. Let us present two examples:

Valeria Ferrari [2] derived the following formulae; $P=\frac{32}{5} \frac{G^{4}}{c^{5}} \frac{\mu^{2} M^{3}}{l_{0}^{5}}[\mathrm{~W}]$, where $\mu=\frac{m_{1} m_{2}}{M}, M=m_{1}+m_{2}$ and $l_{0}=2 \rho_{0}$ is the orbital separation. If $m_{1}=m_{2}$, we get

$$
P=\frac{2}{5} \frac{G^{4}}{c^{5}} \frac{m^{5}}{\rho_{0}^{5}}[\mathrm{~W}]
$$

In the book of Gasperini [3], we find $P=\frac{32}{5} \frac{G}{c^{2}} a^{4} \omega_{0}^{6}[\mathrm{~W}]$, where $a$ is wrongly defined as one half of the orbital separation. Deleting this error and insertion 


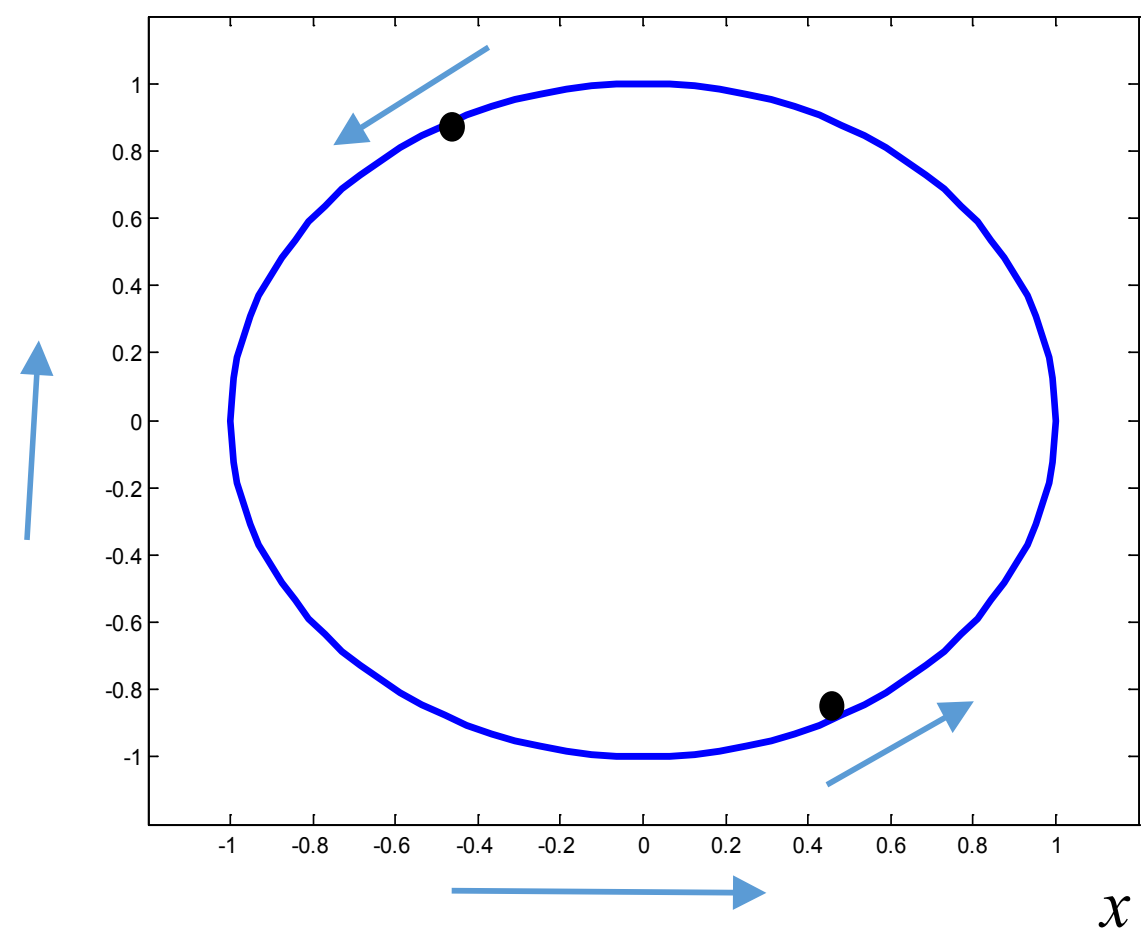

Figure 1. The circular orbit of binary stars. Cartesian coordinates $(x, y)$.

the formula (2), we get again (3). The above presented well known theory does not explain the phenomenon of inspiral. The famous observations by Taylor and Hulse [4] [5] [6], of the binary pulsar PSR1913+16 have shown that the stars inspiral. The instantaneous radius $\rho(t)$ decrease and the instantaneous angular velocity $\omega(t)$ increase. In order to explain this phenomenon we introduced a description of the circular system using the notion of instantaneous complex frequency [7] [8].

\section{Instantaneous Complex Frequency Description of the Circular Binary System}

We have to show that due to the emission of gravitational waves, the trajectory of the stars is not circular since the instantaneous radius decrease in time and the angular velocity and tangential velocity increase in time. The stars are orbiting along spirals (Figure 2). A convenient method of description of this phenomenon is the notion of instantaneous complex frequency. The phasor representing the first star has the form

$$
\psi_{1}(t)=\rho_{0} \exp \left[\int_{0}^{t} s(t) \mathrm{d} t+j \varphi(0)\right] ; s(t)=\alpha(t)+j \omega(t)
$$

and the second one

$$
\psi_{2}(t)=\rho_{0} \exp \left[\int_{0}^{t} s(t) \mathrm{d} t+j[\varphi(0)+\pi]\right],
$$

$\alpha(t)$ is called instantaneous radial frequency and $\omega(t)$-instantaneous angular frequency. The instantaneous radius is $\rho(t)=\rho_{0} \exp \left[\int_{0}^{t} \alpha(t) \mathrm{d} t\right]$. In the 


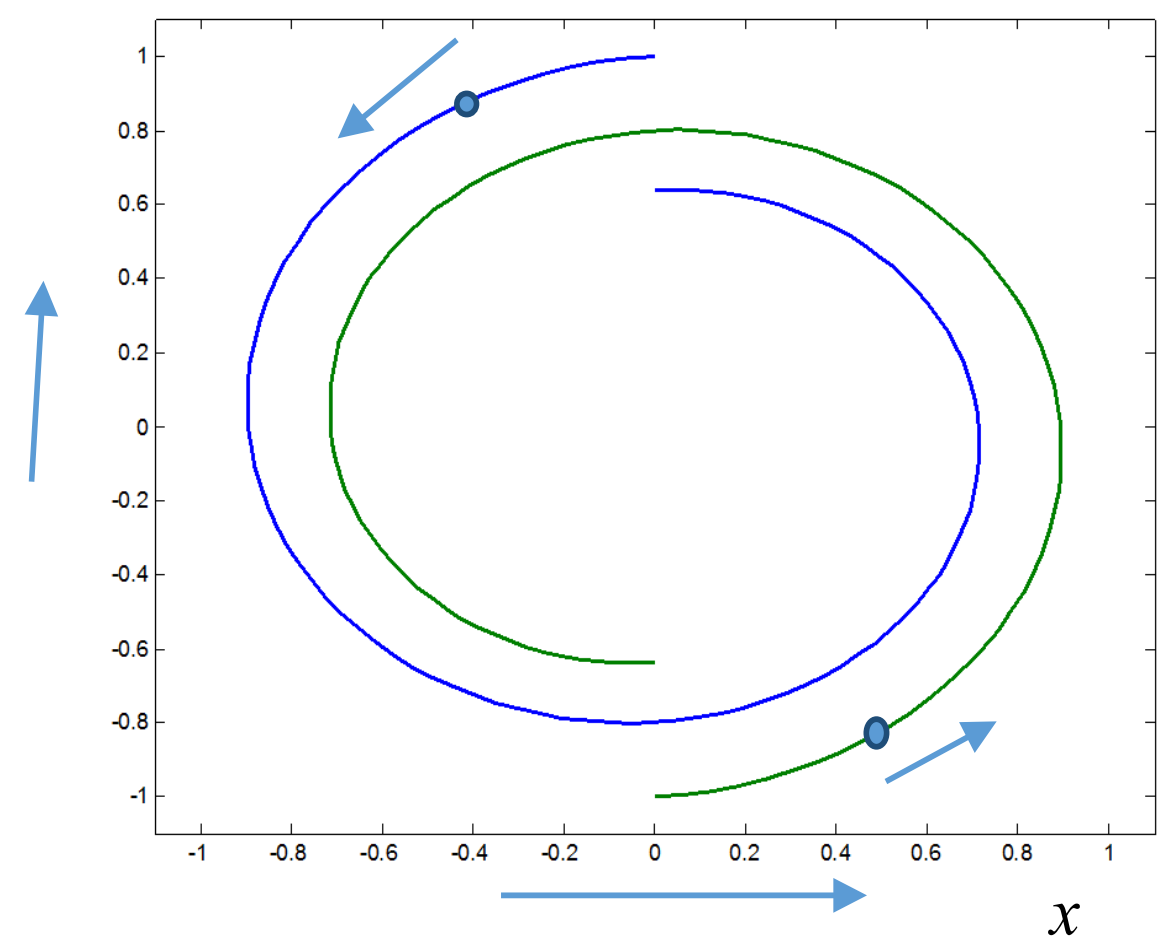

Figure 2. Inspiral orbits with enlarged rate of inspiral of binary stars. Cartesian coordinates $(x, y)$.

simplest description of the properties of inspiral, the instantaneous complex frequency is time independent: $s(t)=-\alpha_{0}+j \omega_{0}$. In this case, the Cartesian coordinates of the orbit are:

○ For the first star $x(t)=\rho_{0} \exp \left(-\alpha_{0} t\right) \cos \left(\omega_{0} t\right)$;

$$
y(t)=\rho_{0} \exp \left(-\alpha_{0} t\right) \sin \left(\omega_{0} t\right) ;
$$

○ For the second $x(t)=-\rho_{0} \exp \left(-\alpha_{0} t\right) \cos \left(\omega_{0} t\right)$;

$y(t)=-\rho_{0} \exp \left(-\alpha_{0} t\right) \sin \left(\omega_{0} t\right)$.

We have to explain why the stars accelerate by orbiting along the inspiral orbit. Let us show that the gravitational force $F_{g}=\frac{G m^{2}}{4 \rho^{2}(t)}$ and the centrifugal force $F_{c}=m \omega_{0}^{2} \rho_{c}$ differ by magnitude and direction. The orbital distance $2 \rho(t)=2 \rho_{0} \exp \left[-\int_{0}^{t} \alpha(t) \mathrm{d} t\right]$ is a line connecting the mass centers through the origin $(0,0)$ and defines the direction of gravitation force. Differently, the direction of the centrifugal force $F_{c}$ is defined by the curvature radius $\rho_{c}$ The geometry of the addition of the two forces is presented in Figure 3 (with large rate of inspiral). In the case $\alpha(t)=-\alpha_{0}$, the angle $\gamma$ is given by the formulae (see Appendix 1)

$$
\begin{aligned}
& \tan (\gamma(t=0))=-\frac{\alpha_{0}}{\omega_{0}}, \sin (\gamma(t=0))=\frac{-\alpha_{0} / \omega_{0}}{\sqrt{1+\left(\alpha_{0} / \omega_{0}\right)^{2}}}, \\
& \cos (\gamma(t=0))=\frac{1}{\sqrt{1+\left(\alpha_{0} / \omega_{0}\right)^{2}}} .
\end{aligned}
$$



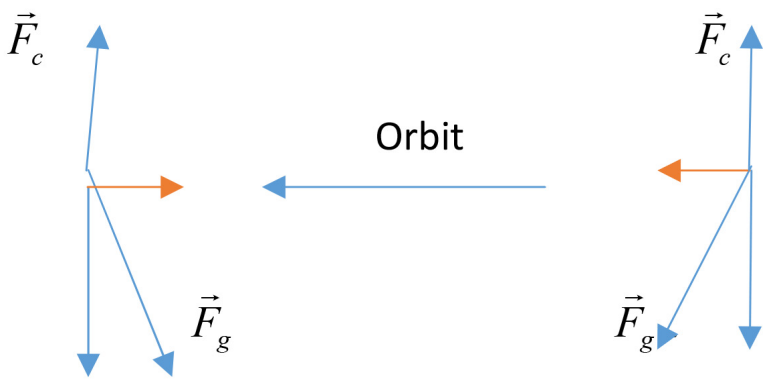

\section{De-acceleration}

Acceleration

Figure 3. The gravitation force $\boldsymbol{F}_{g}$ is not collinear with the centripetal force $\boldsymbol{F}_{c}$ the forces $\boldsymbol{F}_{c}$ and $\boldsymbol{F}_{g} \cos (\gamma)$ cancel and $\boldsymbol{F}_{g} \sin (\gamma)$ has the direction of the tangent of the orbit causing de-acceleration (right) or deceleration (left).

The inspiral orbit is defined by the equation

$$
\text { Gravitational force } \times \cos [\gamma(t)]=\text { Centrifugal force } .
$$

However, there is a tangential force (see Figure 3)

$$
\text { Tangential force }=F_{\text {tan }}=\text { Gravitational force } \times \sin [\gamma(t)] .
$$

However, for the quasi-circular orbit, the tangential force is extremely small. This force induces acceleration of the mass $m$ given by

$$
a(t)=m \boldsymbol{F}_{\tan } .
$$

In consequence, the instantaneous angular frequency is increasing in time

$$
\omega(t)=\omega_{0}+\int_{0}^{t} a(t) \mathrm{d} t=\frac{2 \pi}{T(t)}
$$

where $T(t)$ is a decreasing instantaneous period. The energies of the system also increase in time. The instantaneous tangential velocity of the stars is

$$
v(t)=\omega(t) \rho_{c}(t) .
$$

The curvature radius is (see Appendix 2)

$$
\rho_{c}(t)=\rho_{0} \mathrm{e}^{-\alpha_{0} t} \frac{\left(1+\left(\alpha_{0} / \omega_{0}\right)^{2}\right)^{3 / 2}}{1-\left(\alpha_{0} / \omega_{0}\right)^{2}} .
$$

The instantaneous kinetic energy of both stars is $E_{k}(t)=m v^{2}(t)$ and the instantaneous negative potential energy is $E_{p}(t)=F_{g} \rho(t)=-\frac{G m^{2}}{4 \rho(t)}$. We start the investigations with Equation (2). We can define the value of the radius $\rho_{0}$ or of $\omega_{0}$ but not of both. Our choice is the value $\omega_{0}=2 \pi / T_{0}=2.259554 \times 10^{-4}[\mathrm{rad} / \mathrm{s}]$; $T_{0}=2.788720 \times 10^{4}[\mathrm{~s}]$ measured by Taylor and Hulse [8]. Using (2), we get the following values: the radius $\rho_{0}=9.706500 \times 10^{8}[\mathrm{~m}]$. The tangential velocity equals $v=\omega_{0} \rho_{0}=2.193236 \times 10^{6}[\mathrm{~m} / \mathrm{s}]$ (about $219 \mathrm{~km} / \mathrm{s}$ ). The kinetic energy of both stars is $E_{k}=m v^{2}=1.346134 \times 10^{41}[\mathrm{~J}]$ and the negative potential energy 
(magnitude twice of $E_{k}$ ) is $E_{p}=-G m^{2} /\left(2 \rho_{0}\right)=-2.692268 \times 10^{41}[\mathrm{~J}]=-2 E_{k}$. The total energy of the system is negative. The power of the gravitational waves emitted by the system given by Equation (3) is $P=6.523698 \times 10^{23}[\mathrm{~W}]$.

1) Estimation of the value of the radial frequency $\alpha_{0}$

The decrease of the radius of the circular model in one period $T_{0}$ is

$$
\rho_{1}=\rho_{0} \mathrm{e}^{-\alpha_{0} T_{0}}=\rho_{0} \mathrm{e}^{-2 \pi\left(\alpha_{0} / \omega_{0}\right)} .
$$

We have an increase of the negative value of the potential energy

$$
E_{p 1}=-\frac{G m^{2}}{4 \rho_{1}} \approx-\frac{G m}{4 \rho_{0}}\left(1+2 \pi \alpha_{0} / \omega_{0}\right) .
$$

Therefore, we get the increase

$$
\nabla E_{p 1} \approx \frac{-G m}{4 \rho_{0}}\left(2 \pi \alpha_{0} / \omega_{0}\right) .
$$

Assuming arbitrary that this increase should be equal to the energy emitted by gravitational waves during one period we get

$$
\nabla E_{p 1} \approx \frac{-G m}{4 \rho_{0}}\left(2 \pi \alpha_{0} / \omega_{0}\right)=P T_{0}=P 2 \pi / \omega_{0} .
$$

Therefore,

$$
\alpha_{0} \approx-\frac{P}{E_{p}}\left[\mathrm{~s}^{-1}\right]=-6.526980 \times 10^{23} / 2.692268 \times 10^{41}=-2.424343 \times 10^{-18} .
$$

2) The increase of the angular frequency (or decrease of the period $T_{0}$ )

\section{during the inspiral}

Taylor and Hulse have measured that the period of the PSR system decreases by $76.5 \mu$ s per year [6]. Let us derive a formula for this decrease for the circular system. We insert in Equation (2) $\rho(t)=\rho_{0} \mathrm{e}^{-\alpha_{0} t}$ in place of $\rho_{0}$ getting

$$
\omega(t)=\sqrt{\frac{G m}{4 \rho_{0}^{4} \mathrm{e}^{-3 \alpha_{0} t}}}=\omega_{0} \mathrm{e}^{\frac{3}{2} \alpha_{0} t} \rightarrow T(t)=T_{0} \mathrm{e}^{-\frac{3}{2} \alpha_{0} t} .
$$

The decrease of the period per year is

$$
\nabla T_{\text {year }}=T_{0}-T\left(t_{\text {year }}\right)=T_{0}\left[1-\mathrm{e}^{-\frac{3}{2} \alpha_{0} t_{\text {year }}}\right] \approx-\frac{3}{2} T_{0} \alpha_{0} t_{\text {year }}=3.18022 \times 10^{-6}[\mathrm{~s}] .
$$

It is more than one order of magnitude smaller in comparison to $76,5 \mu \mathrm{s}$ per year of the PSR system. Therefore, the circular model cannot be applied to describe the properties of the PSR elliptical system.

3) The increase of the negative value of the potential energy

The potential energy at the moment $t=0$ is

$$
E_{p}(0)=-\frac{G m^{2}}{2 \rho_{0}}=-2.692268 \times 10^{41}[\mathrm{~J}] .
$$

The value after one year is

$$
E_{p}\left(t_{\text {year }}\right)=-\frac{G m^{2}}{2 \rho_{0} \mathrm{e}^{-\alpha_{0} t_{\text {year }}}}[\mathrm{J}] .
$$


The increase is

$$
\nabla E_{p}=E_{p}(0)\left(\mathrm{e}^{\alpha_{0} t_{y} \text { yar }}-1\right)=-2.052708 \times 10^{31}[\mathrm{~J}] .
$$

The division by $t_{\text {year }}$ yields the power

$$
P=\frac{\nabla E_{p}}{t_{\text {year }}}=-6.526917 \times 10^{23}[\mathrm{~W}],
$$

i.e., exactly the value defined by Equation (3) which represents the power of the emitted gravitational waves. This result validates the correctness of Equation (17) defining $\alpha_{0}$ and Equation (19) defining the delay per year. The negative sign of this power is applied in the book of Gasperini [3] with no comment. We found that the authors of reference [9] derived a formula with a negative sign of the gravitational "Poynting vector" also with no comment.

\section{4) The inspiral time}

The main goal of this paper is to validate the explanation of the nature of gravity presented in [1]. Having this in mind, let us describe only briefly the process of inspiral. During each period the radius $\rho(t)=\rho_{0} \exp \left(-\int_{0}^{t} \alpha(t) \mathrm{d} t\right)$ is a bit shorter, each next period is shorter corresponding to an increase of the angular frequency which is a function of time. As well, the instantaneous radial frequency increases with time. The angular frequency is

$$
\omega(t)=\sqrt{\frac{G m^{2}}{4 \rho^{3}(t)}}=\omega_{0} \mathrm{e}^{\frac{3}{2} \alpha_{0} t} ; \quad \alpha(t)=\alpha_{0} .
$$

We get

$$
T(t)=T_{0} \mathrm{e}^{-\frac{3}{2} \alpha_{0} t} \text { and } \nabla T(t)=T_{0}-T(t)=T_{0}\left[1-\mathrm{e}^{-\frac{3}{2} \alpha_{0} t}\right] \approx \frac{-3}{2} T_{0} \alpha_{0} t .
$$

The instantaneous radius is

$$
\rho(t)=\rho_{0} \exp \left[-\int_{0}^{t} \alpha(t) \mathrm{d} t\right] \approx \rho_{0} \mathrm{e}^{-\alpha_{0} t} ; \alpha(t) \approx \alpha_{0} .
$$

The decrease of the radius during a year is

$$
\nabla \rho_{\text {year }}=\rho_{0}\left(1-\mathrm{e}^{-\alpha_{0} t_{\text {year }}}\right) .
$$

The overestimated number of years of the total inspiral (overestimated since calculated using the constant value $\alpha_{0}$ ) is

$$
\text { Number of years }=\frac{\rho_{0}}{\nabla \rho_{\text {year }}}=\frac{1}{1-\mathrm{e}^{-\alpha_{0} t_{\text {year }}}} \approx \frac{\sim 1}{\alpha_{0} t_{\text {year }}}=1.098 \times 10^{10}[\text { years }] .
$$

\section{5) Concluding remarks about the circular system}

During a single revolution, the emitted power may be classified as time independent since the increase is negligible. The directional pattern $\sigma(\Omega)[\mathrm{W} /$ steradian] is circular symmetric with maximum radiation in the plane of the circle. The total energy of the system during a single revolution has a negligible time dependence. The radiation is emitted in twice the orbital fre- 
quency with a circular polarization [2]. In long times, there is an increase of the power and the negative energy of the system. Therefore, assuming that the gravitational waves carry positive energy, the emission is at the cost of increasing negative energy of the system. The presented theory of inspiral is valid only in the frame of a linear gravitation. The phenomena in the last stage of inspiral are certainly governed by nonlinear effects. Recently, the LIGO system registered a chirp like signal of duration about 0.17 [s] emitted by two black holes shortly before the collapse [10] [11]. Certainly, a linear theory is unable to describe this signal.

\section{The Theoretical Model of the Binary Pulsar PSRB1913+16}

The PSR system differs considerably from the above described circular system. The two stars are orbiting along elliptical orbits (see Figure 4) around a common center of mass located in the focus. We consider again equal masses $m_{1}=m_{2}=m$. The data of this system measured by Taylor and Hulse are presented in Appendix 1. In the circular model, the distance between the stars, the angular velocity $\omega$, the tangential velocity and the potential and kinetic energies could be classified as constant during a single orbital time. This is not the case in the PSR system. For example, the velocity changes from $v_{\max }=450[\mathrm{~km} / \mathrm{s}]$ to 120 $[\mathrm{km} / \mathrm{s}]$. These changes overshadow by several orders the inspiral change due to the emission of gravitational waves.

1) The description of the elliptical orbits in polar coordinates centered at the focus

The elliptical orbit of the first star can be defined in polar coordinates $(\rho, \varphi)$ centered at the right focus of the left ellipse (Figure 4). The radius $\rho$ in terms of the angle $\varphi$ is given by the formula

$$
\rho(\varphi)=a \frac{1-\varepsilon^{2}}{1+\varepsilon \cos (\varphi)}
$$

and for the second one centered in the left focus of the right ellipse is

$$
\rho(\varphi)=a \frac{1-\varepsilon^{2}}{1-\varepsilon \cos (\varphi+\pi)}
$$

Note that the angle $\varphi$ is a function of time. However, our derivations have the form of functions of $\varphi$. The inverse function $t(\varphi)$ has no closed form. Our goals do not require the presentation of these relations.

For $\varphi=0$, we get periastrone separation $=2 a[1-\varepsilon]$ and for $\varphi=\pi$, the apastrone separation $=2 a[1+\varepsilon]$. The insertion of the semi-major axis $a=9.7506 \times 10^{8}[\mathrm{~m}]$ and eccentricity $\varepsilon=0.617733$ yields (see [6]):

Periastron separation $=7.45466 \times 10^{8}[\mathrm{~m}]$ and Apastron separation $=3.154477 \times 10^{8}[\mathrm{~m}]$.

2) Why the stars accelerate and decelerate?

In the previous section, we explained why the stars on a circular orbit accelerate. For a circular orbit, this acceleration is extremely small. Differently, in the 


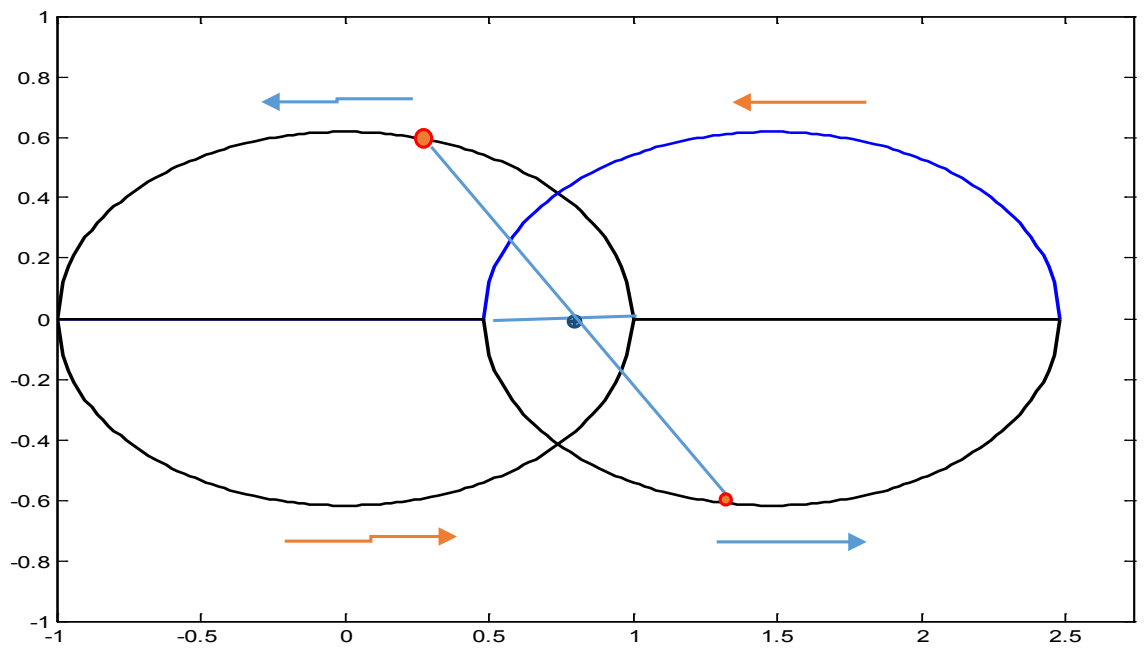

Figure 4. The elliptical orbits of the PSR system. The stars (red points) rotate anti clock. Blue arrows: deceleration. Red arrows: acceleration.

case of the two elliptical orbits we have large accelerations and decelerations during each period. Again, the gravitational attraction force is given by

$$
\boldsymbol{F}_{g}(\varphi)=\frac{G m^{2}}{4 \rho^{2}(\varphi)}[\mathrm{N}]
$$

and the centrifugal force is given by

$$
\boldsymbol{F}_{c}(t)=m \omega^{2}(\varphi) \rho_{c}(\varphi)[\mathrm{N}]
$$

where $\rho_{c}$ is the curvature radius of the ellipse and the angular velocity is defined by the rotation of the curvature radius. Again, the force vectors have different direction defined by the angle $\gamma$. The gravitational force can be represented by a vector sum of two perpendicular terms (Figure 3). The term

$$
\boldsymbol{F}_{g}(\varphi) \cos (\gamma)=\frac{G m^{2}}{4 \rho^{2}(\varphi)} \cos (\gamma)[\mathrm{N}]
$$

has the direction of (30) perpendicular to the tangent of the ellipse and the term

$$
\boldsymbol{F}_{g}(\varphi) \sin (\gamma)=\frac{G m^{2}}{4 \rho^{2}(\varphi)} \sin (\gamma)[\mathrm{N}]
$$

represents a tangential force. Equating the terms (31) and (32) yields the following formula for the local angular velocity (local means the function of $\varphi$ ).

$$
\omega(\varphi)=\sqrt{\frac{G m \cos (\gamma)}{4 \rho(\varphi) \rho_{c}(\varphi)}}[\mathrm{rad} / \mathrm{s}] .
$$

The local tangential acceleration is

$$
a(\varphi)=\boldsymbol{F}_{g}(\varphi) \sin (\gamma) / m=\frac{G m}{4 \rho^{2}(\varphi)} \sin (\gamma)\left[\mathrm{m} / \mathrm{s}^{2}\right] .
$$

The local velocity of the stars by orbiting from periastrone to apastron is (deceleration) 


$$
v(\varphi)=v_{\max }-\int_{0}^{\pi} a(\varphi) \mathrm{d} \varphi
$$

and in opposite direction (acceleration)

$$
v(\varphi)=v_{\min }+\int_{\pi}^{2 \pi} a(\varphi) \mathrm{d} \varphi .
$$

The mean velocity (in terms of $\varphi$ ) is the same for both directions

$$
v_{\text {mean }}=\frac{1}{\pi} \int_{0}^{\pi} v(\varphi) \mathrm{d} \varphi .
$$

The local tangential velocity is alternatively defined as

$$
v(\varphi)=\omega(\varphi) \rho_{c}(\varphi)
$$

Where $\rho_{c}$ is the local curvature radius (see Figure 5). The local velocity is shown in Figure 6. The maximum value equals $448.172[\mathrm{~km} / \mathrm{s}]$ and the minimum 106.287 (compare with Appendix 1).

Note that in this model, the maxima and minima of the velocity are located near the periastrone and apastrone (not exactly at these locations). The mean value in terms of $\varphi$ (as in Equation (38)) is $269.782[\mathrm{~km} / \mathrm{s}]$. The time average is

$$
\bar{v}(t)=\frac{\text { circumference of ellipse }}{\text { time of a single revolution }}=181.757[\mathrm{~km} / \mathrm{s}] \text {. }
$$

The local average differs from the time average by the factor 1.319 . The local angular velocity is shown in Figure 7.
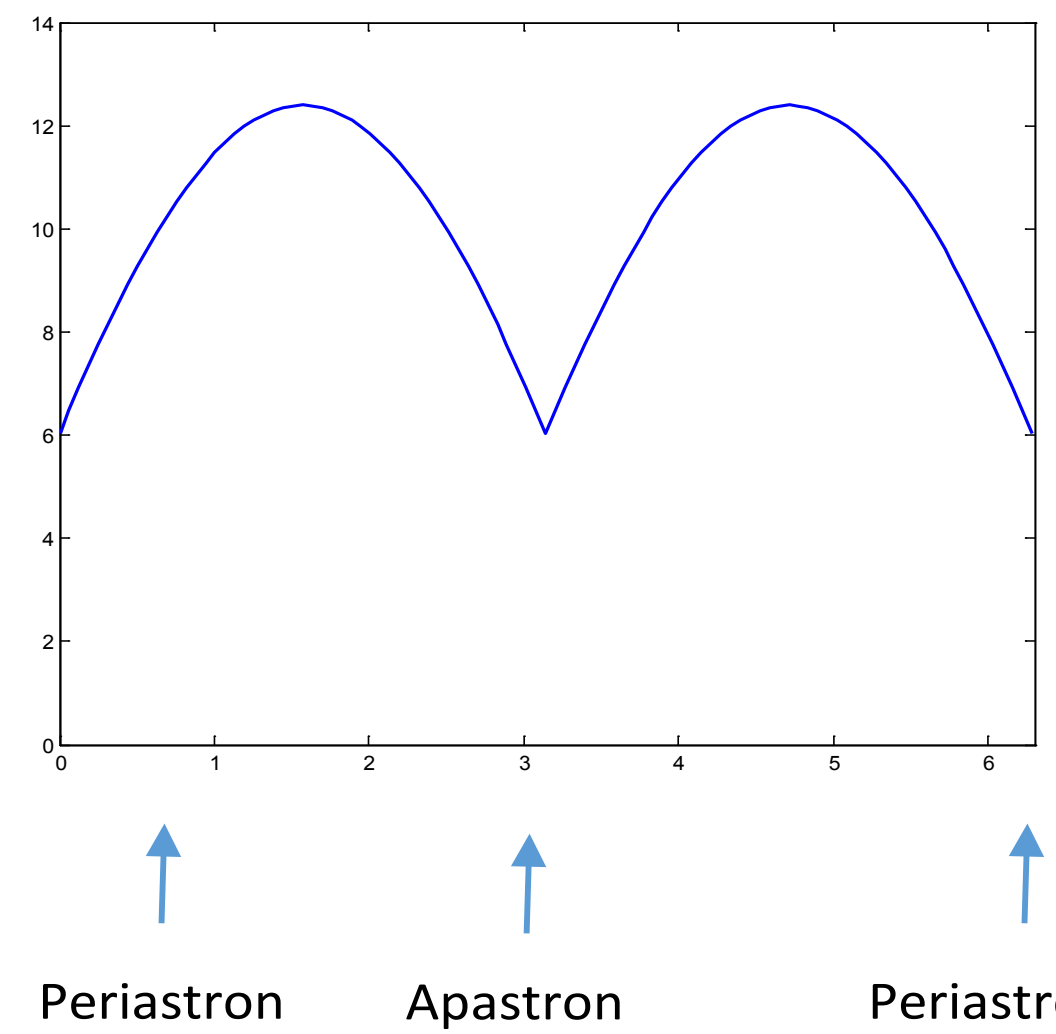

Periastron

Figure 5. Curvature radius in terms of $\varphi$. Vertical scale $\times 10^{8}$. 


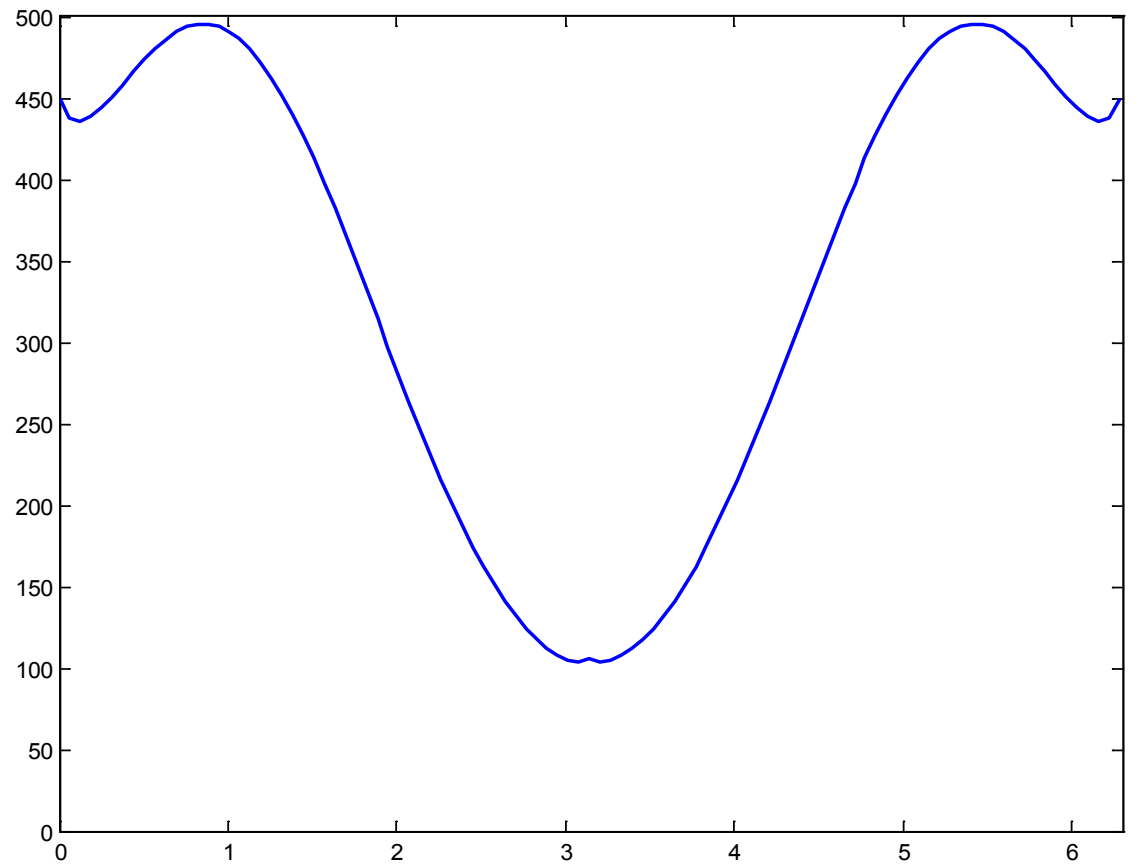

Figure 6. Orbital velocity $[\mathrm{km} / \mathrm{s}]$ in terms of $\varphi$. Note the localization of $v_{\max }$ not at periastron.

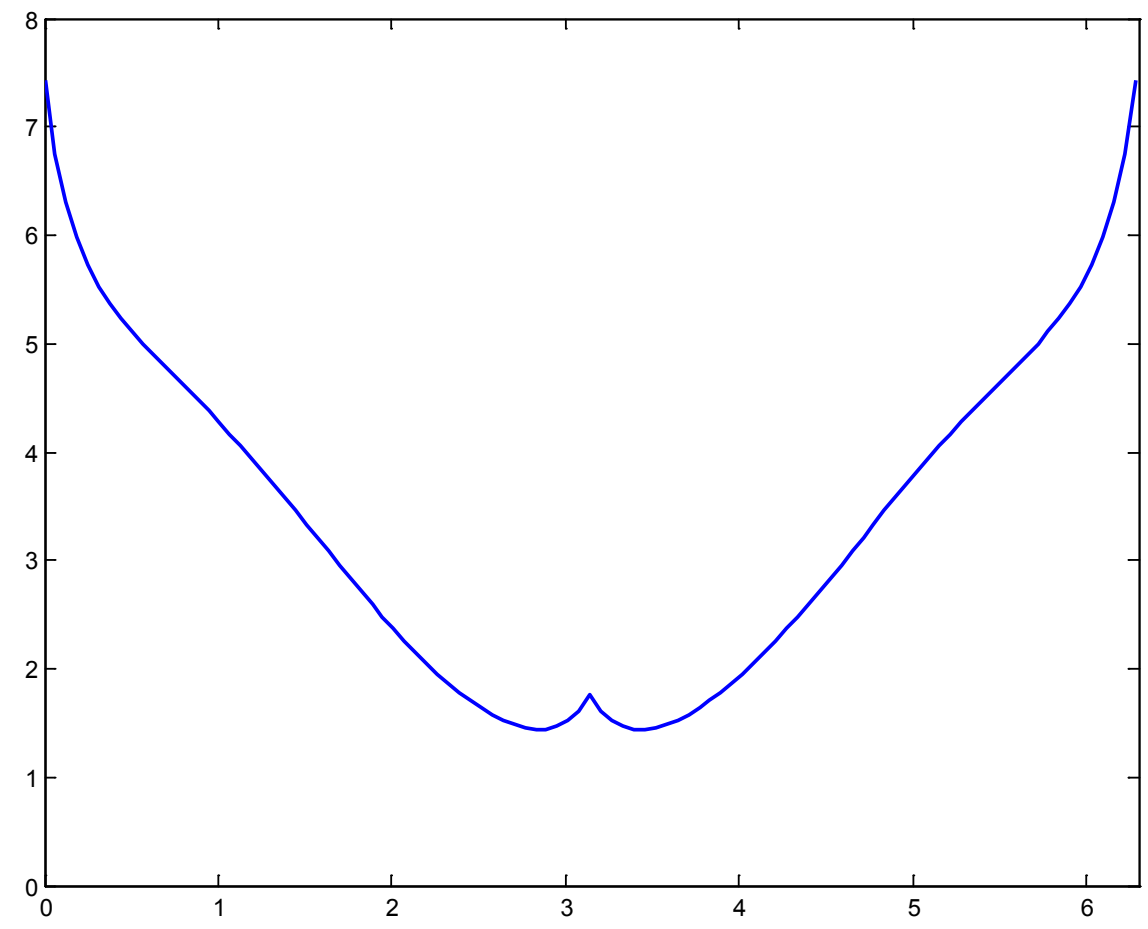

Figure 7. Angular velocity $\omega(\mathrm{rad} / \mathrm{s})$ in terms $\varphi$. Vertical scale $10^{-8}$.

3) Kinetic and potential energies in the PSR system

The local kinetic energy is (Figure 8)

$$
E_{k}(\varphi)=m v^{2}(\varphi)[\mathrm{J}]
$$




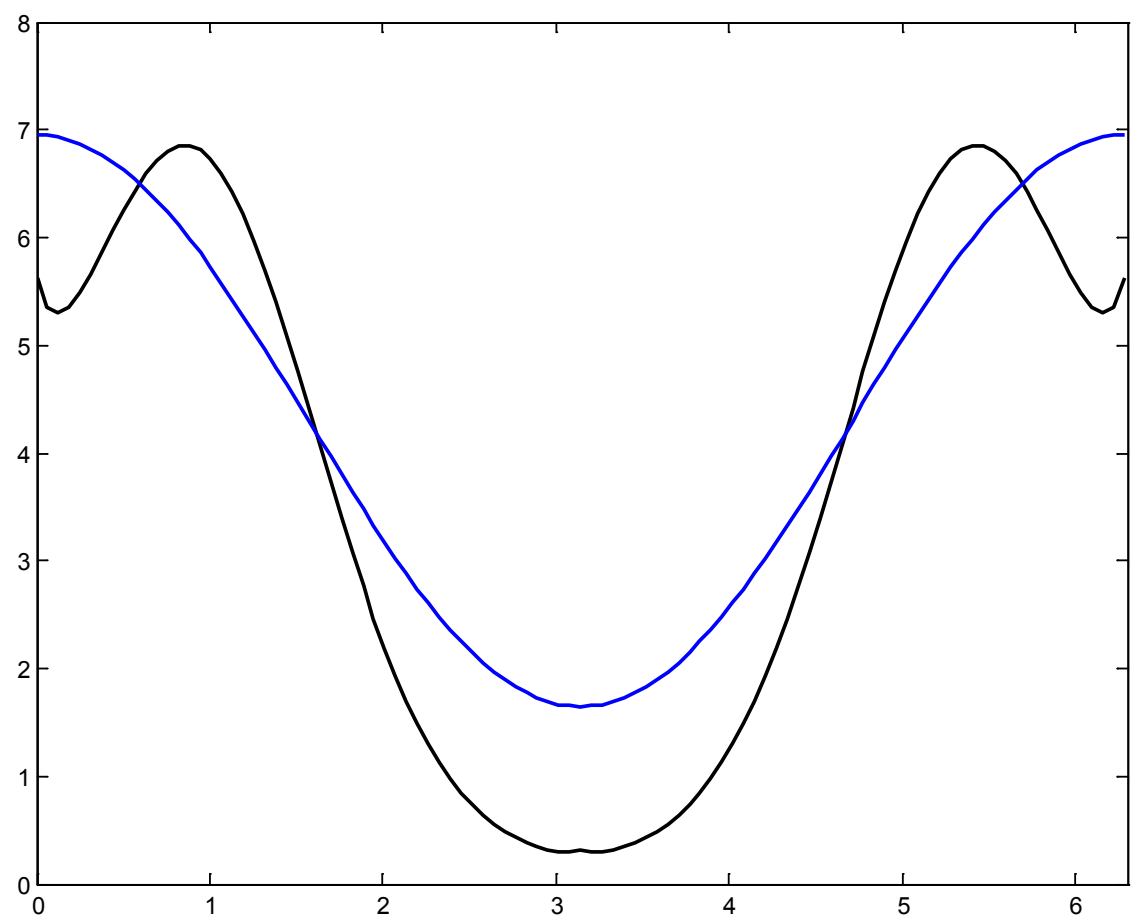

Figure 8. Kinetic energy (upper curve at $\varphi=\pi$ ) and the magnitude of potential energy in terms of $\varphi$. Vertical scale $\times 10^{41}$.

and the local negative potential energy is

$$
E_{P}(\varphi)=\frac{-G m^{2}}{2 \rho(\varphi)}[\mathrm{J}] .
$$

The corresponding local averages are $\bar{E}_{k}=2.38257 \times 10^{41}[\mathrm{~J}]$ and $\bar{E}_{p}=-4.369225 \times 10^{41}[\mathrm{~J}]$. The ratio is $\left|\boldsymbol{E}_{p}\right| / E_{k}=2.02435 \approx 2$, i.e., almost the value defined by the circular system. It is reasonable to assume that the same ratio is valid for time averages.

\section{4) Concluding remarks about the PSR system}

Differently to the circular system the power emitted during a single revolution is a function of time and the radiation pattern $\sigma(\Omega)[\mathrm{W} /$ steradian] is a periodic function of time. Therefore, the reported by Taylor and Hulse power of the emitted gravitational waves $\mathrm{P}=7.35 \times 10^{24}[\mathrm{~W}]$ should be classified as a mean value.

\section{Final Conclusions}

- Both orbits, the circular and the elliptical, are defined by two forces of opposite directions: the centrifugal force and a term of the gravitational force (see Figure 3). It is logical to assume that both forces have the same physical explanation: the anisotropic energy exchange as described in reference [1]. Here, both anisotropies of radiation cancel. The other part of the gravitational force responsible for tangential acceleration or deceleration is the result of the tangential anisotropy of radiation. 
- In the case of a circular orbit, the tangential acceleration is extremely small. We have shown that the notion of the instantaneous complex frequency is a convenient tool to study the process of inspiral of circular systems in the range of a linear gravitational force.

Arguments in favor of the radiation recoil nature of gravity presented in [1 m]

In 2015, the author presented a paper "Gravitational Forces Explained as the Result of Exchange between Baryonic Matter and the Quantum Vacuum" [1]. Let us present arguments in favor of this theory in terms of the description of orbiting double stars given in this paper:

o The orbit is defined by two forces: the gravitational force directed towards inside of the orbit and the centrifugal force directed towards the outside of the orbit. The forces are not collinear.

- The centripetal force is perpendicular to the tangent of the orbit.

- The gravitational force except some points is not perpendicular to the tangent and can be decomposed in two terms: the perpendicular compensates the centripetal force. The two forces cancel.

- The tangent force is responsible for acceleration or deceleration of the star.

- In the case of the common nearly circular orbit of the stars, we have an extremely small acceleration. In the case of a double-elliptical orbit, we have large accelerations and deceleration.

o The cancelation of the two forces shows that gravity and inertia have the same physical origin. They are recoil forces of radiation. The radiation pattern should be symmetric w.r.t. the tangent of the orbit. Differently, the pattern is asymmetric w.r.t. the line perpendicular to the orbit resulting in a recoil force of radiation.

In a word, it is logical to assume that all described here forces are recoil forces of radiation. The radiation pattern is symmetric w.r.t. the tangent of the orbit (cancellation of gravitation and inertia) and asymmetric w.r.t. the line perpendicular to the orbit, i.e., the direction of the curvature radius.

\section{Conflicts of Interest}

The authors declare no conflicts of interest regarding the publication of this paper.

\section{References}

[1] Hahn, S.L. (2015) Journal of Modern Physics, 6, 1135-1145. https://doi.org/10.4236/jmp.2015.68117

[2] Ferrari, V. (2010) The Quadrupole Formalism Applied to Binary Systems. https://www.-ego-gw.it/public/events/vesf

[3] Gasperini, M. (2017) Theory of Gravitational Interactions. 2nd Edition, Springer.

[4] Press Release (1993) About the Nobel Prize in Physics, Royal Swedish Academy of Sciences. 
[5] The Binary Pulsar PSR1913+16.

http://www.astrocornell.edu/academics/PSR1913+16

[6] Johnstone, R. http://www.johnstonsarchive.net./relativity/binarypulsar.html

[7] Hahn, S. (1939) The Instantaneous Complex Frequency Concept and Its Application in the Analysis of Building up Oscillations in Oscillator. Proceedings of Vibrations Problems, No. 1, 29-46.

[8] Hahn, S.L. and Snopek, K.M. (2016) Complex and Hypercomplex Analytic Signals. Theory and Applications, Artech House, Boston-Landon.

[9] Mironov, V.L. and Mironov, S.V. (2014) Journal of Modern Physics, 5, 917-929. https://doi.org/10.4236/jmp.2014.510095

[10] The 2017 Nobel Prize in Physics-Press Release=Nobelprize.org.

[11] https://www.nobelprize.org/nobel_prizes/physics/laureates/2917/pr 


\section{Appendix 1}

This paper is illustrated by the properties of the binary pulsar PSR1913+16, a system of two binary neutron stars discovered and measured during many years by Taylor and Hulse [7] [8]. This great achievement of radioastronomy and also of time-frequency metrology was awarded by the Nobel Prize in physics in 1993. Let us repeat here the data compiled by Robert Johnston [8].

Mass of detected pulsar $m_{1}=1.441 \times$ solar mass $=2.8764 \times 10^{30}[\mathrm{~kg}]$ and of its companion $m_{2}=1.387 \times$ s.m. $=2.7205 \times 10^{30}[\mathrm{~kg}]$.

Orbital period $T_{0}=7.7511939106[\mathrm{hr}]=27807.19557[\mathrm{~s}]$.

Eccentricity of the elliptical orbits $\varepsilon=0.617131$.

Semi-major axis $2 a=1950100[\mathrm{~km}]$ (remark of this author: the name semi-major axis should be replaced by major axis. Semi-major axis is equal not $2 a$ but a. Periastron separation $=746600[\mathrm{~km}]$, Apastron separation $=3153600[\mathrm{~km}]$

Orbital velocity of stars relative to the center of mass: at periastrone 450 $[\mathrm{km} / \mathrm{s}]$, at apastrone: $110[\mathrm{~km} / \mathrm{s}]$.

\section{Appendix 2: The Derivation of the Curvature Radius of the in Spiral Orbit}

Let us define the in spiral orbit by the equation

$$
\begin{gathered}
\psi(t)=\rho_{0} \mathrm{e} \int_{0}^{t} s(t) \mathrm{d} t \\
s(t)=-(\alpha+\Delta \alpha * t)+j\left(\omega_{0}+\Delta \omega * t\right)
\end{gathered}
$$

We assume that the binary stars have equal mass and rotate synchronously around common center of mass located at $x=y=0$. The Cartesian coordinates of the first star are defined by the complex function $\psi(t)=x(t)+j y(t)$ with

$$
\begin{aligned}
& x(t)=\rho_{0} \mathrm{e}^{-\left(\alpha t+0.5 \Delta \alpha * t^{2}\right)} \cos \left(\omega_{0} t+0.5 \Delta \omega * t^{2}\right) \\
& y(t)=\rho_{0} \mathrm{e}^{-\left(\alpha t+0.5 \Delta \alpha * t^{2}\right)} \sin \left(\omega_{0} t+0.5 \Delta \omega * t^{2}\right)
\end{aligned}
$$

and for the second star

$$
\begin{aligned}
& x(t)=-\rho_{0} \mathrm{e}^{-\left(\alpha t+0.5 \Delta \alpha * t^{2}\right)} \cos \left(\omega_{0} t+0.5 \Delta \omega * t^{2}\right) \\
& y(t)=-\rho_{0} \mathrm{e}^{-\left(\alpha t+0.5 \Delta \alpha t^{2}\right)} \sin \left(\omega_{0} t+0.5 \Delta \omega * t^{2}\right)
\end{aligned}
$$

The curvature radius at the point defined by $t=t_{0}$ is

$$
\rho_{c}=\frac{\left[\left(\dot{x}\left(t_{0}\right)^{2}\right)+\left(\dot{y}\left(t_{0}\right)^{2}\right)\right]}{\left|\ddot{y}\left(t_{0}\right) \dot{x}\left(t_{0}\right)+\ddot{x}\left(t_{0}\right) \dot{y}\left(t_{0}\right)\right|}
$$

Let us calculate the derivatives beginning with zero values of $\Delta \alpha$ and $\Delta \omega$. We have

$$
\dot{x}(t)=\rho_{0}\left[-\alpha_{0} \mathrm{e}^{-\alpha_{0} t} \cos \left(\omega_{0} t\right)-\omega_{0} \mathrm{e}^{-\alpha_{0} t} \sin \left(\omega_{0} t\right)\right]
$$




$$
\begin{aligned}
\dot{y}(t)= & \rho_{0}\left[-\alpha_{0} \mathrm{e}^{-\alpha_{0} t} \sin \left(\omega_{0} t\right)+\mathrm{e}^{-\alpha_{0} t} \omega_{0} \cos \left(\omega_{0} t\right)\right] \\
\ddot{x}(t)= & \rho_{0}\left[\alpha_{0}^{2} \mathrm{e}^{-\alpha_{0} t} \cos \left(\omega_{0} t\right)+\alpha_{0} \omega_{0} \mathrm{e}^{-\alpha t} \sin \left(\omega_{0} t\right)\right. \\
& \left.+\alpha_{0} \omega_{0} \mathrm{e}^{-\alpha t} \sin \left(\omega_{0} t\right)-\omega_{0}^{2} \mathrm{e}^{-\alpha_{0} t} \cos \left(\omega_{0} t\right)\right] \\
\ddot{y}(t)= & R_{0}\left[\alpha_{0}^{2} \mathrm{e}^{-\alpha_{0} t} \sin \left(\omega_{0} t\right)-\alpha_{0} \omega_{0} \mathrm{e}^{-\alpha_{0} t} \cos \left(\omega_{0} t\right)\right. \\
& \left.-\alpha_{0} \omega_{0} \mathrm{e}^{-\alpha_{0} t} \cos \left(\omega_{0} t\right)-\omega_{0}^{2} \mathrm{e}^{-\alpha t} \sin \left(\omega_{0} t\right)\right]
\end{aligned}
$$

The insertion using $t=0$ yields

$$
\rho_{c}(t)=\rho_{0} \mathrm{e}^{-\alpha_{0} t} \frac{\left(\alpha_{0}^{2}+\omega_{0}^{2}\right)^{\frac{3}{2}}}{\left|\omega_{0}\left(\omega_{0}^{2}-\alpha_{0}^{2}\right)\right|}=\rho_{0} \mathrm{e}^{-\alpha_{0} t} \frac{\left(1+\left(\alpha_{0} / \omega_{0}\right)^{2}\right)^{\frac{3}{2}}}{1-\left(\alpha_{0} / \omega_{0}\right)^{2}}
$$

Of course, for a circular orbit $\alpha_{0}=0$ and $\rho_{c}=\rho_{0}$. Otherwise, $\rho_{c}>\rho_{0}$. The center of the curvature radius is located at

$$
\begin{aligned}
& x_{c}=x\left(t_{0}\right)-\dot{y}\left(t_{0}\right) \frac{\left(\left(\dot{x}\left(t_{0}\right)\right)\right)^{2}+\left(\dot{y}\left(t_{0}\right)\right)^{2}}{\ddot{y}\left(t_{0}\right) \dot{x}\left(t_{0}\right)-\dot{y}\left(t_{0}\right) \ddot{x}\left(t_{0}\right)} ; \\
& y_{c}=y\left(t_{0}\right)+\dot{x}\left(t_{0}\right) \frac{\left(\left(\dot{x}\left(t_{0}\right)\right)\right)^{2}+\left(\dot{y}\left(t_{0}\right)\right)^{2}}{\ddot{y}\left(t_{0}\right) \dot{x}\left(t_{0}\right)-\dot{y}\left(t_{0}\right) \ddot{x}\left(t_{0}\right)} .
\end{aligned}
$$

If $t=0, x(0)=\rho_{0}, y(0)=0, \dot{x}(0)=-\alpha_{0} \rho_{0}, \dot{y}(0)=\omega_{0} \rho_{0}$, $\ddot{x}(0)=\rho_{0}\left(\alpha_{0}^{2}-\omega_{0}^{2}\right), \ddot{y}(0)=-2 \alpha_{0} \omega_{0} R_{0}$.

The insertion yields $x_{c}(0)=0, y_{c}(0)=-\frac{\alpha_{0}}{\omega_{0}} \rho_{0}$. The angle between $\rho_{0}$ and $\rho_{c}$ is

$$
\begin{aligned}
& \tan (\varphi(t=0))=-\frac{\alpha_{0}}{\omega_{0}}, \sin (\varphi(t=0))=\frac{\alpha_{0} / \omega_{0}}{\sqrt{1+\left(\alpha_{0} / \omega_{0}\right)^{2}}}, \\
& \cos (\varphi(t=0))=\frac{1}{\sqrt{1+\left(\alpha_{0} / \omega_{0}\right)^{2}}}
\end{aligned}
$$

The curvature radius of an ellipse in Cartesian coordinates $(x, y)$ is $\rho_{c}=a^{2} b^{2}\left(\frac{x^{2}}{a^{4}}+\frac{y^{2}}{b^{4}}\right)^{\frac{3}{2}}$. 